\title{
Spin excitations in a monolayer scanned by a magnetic tip
}

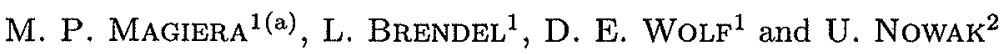 \\ ${ }^{1}$ Department of Physics and CeNIDE, University of Duisburg-Essen - D-47048 Duisburg, Germany, EU \\ 2 Theoretical Physics, University of Konstanz - D-78457 Konstanz, Germany, EU
}

\begin{abstract}
PACS 68.35.Af - Atomic scale friction
PACS $75.10 . \mathrm{Hk}$ - Classical spin models

PACS 75.70.Rf - Surface magnetism
\end{abstract}

\begin{abstract}
Energy dissipation via spin excitations is investigated for a hard ferromagnetic tip scanning a soft magnetic monolayer. We use the classical Heisenberg model with Landau-LifshitzGilbert (LLG) dynamics including a stochastic field representing finite temperatures. The friction force depends linearly on the velocity (provided it is small enough) for all temperatures. For low temperatures, the corresponding friction coefficient is proportional to the phenomenological damping constant of the LLG equation. This dependence is lost at high temperatures, where the friction coefficient decreases exponentially. These findings can be explained by properties of the spin polarisation cloud dragged along with the tip.
\end{abstract}

Copyright (C) EPLA, 2009

Introduction. - While on the macroscopic scale the phenomenology of friction is well known, several new aspects are currently being investigated on the micron and nanometer scale $[1,2]$. During the last two decades, the research on microscopic friction phenomena has advanced enormously, thanks to the development of Atomic Force Microscopy (AFM, [3]), which allows to measure energy dissipation caused by relative motion of a tip with respect to a substrate.

Recently the contribution of magnetic degrees of freedom to energy dissipation processes has attracted increasing interest [4-8]. Today, magnetic materials can be controlled down to the nanometer scale. New developments in the data storage industry, spintronics and quantum computing require a better understanding of tribological phenomena in magnetic systems. For example, the reduction of heat generation in reading heads of hard disks which work at nanometer distances is an important issue, as heat can cause data loss.

Magnetic Force Microscopy (MFM), where both tip and surface are magnetic, is used to investigate surface magnetism and to visualise domain walls. Although recent studies have attempted to measure energy dissipation between an oscillating tip and a magnetic sample $[9,10]$, the dependence of the friction force on the tip's sliding velocity has not been considered yet apart from a work by Fusco et al. [8] which is extended by the present work to temperatures $T \neq 0$. The relative motion of the tip with

\footnotetext{
(a) E-mail: martin.magiera@uni-due.de
}

respect to the surface can lead to the creation of spin waves which propagate inside the sample and dissipate energy, giving rise to magnetic friction.

We will first present a simulation model and define magnetic friction. The model contains classical Heisenberg spins located on a rigid lattice which interact by exchange interaction with each other. Analogous to the reading head of a hard disc or a MFM tip, an external fixed magnetic moment is moved across the substrate. Using Langevin dynamics and damping, it is possible to simulate systems at finite temperatures. The main new results concern the temperature dependence of magnetic friction.

Simulation model and friction definition. - To simulate a solid magnetic monolayer (on a nonmagnetic substrate), we consider a two-dimensional rigid $L_{x} \times L_{y}$ lattice of classical normalised dipole moments ("spins") $\mathbf{S}_{i}=\mu_{i} / \mu_{s}$, where $\mu_{s}$ denotes the material-dependent magnetic saturation moment (typically a few Bohr magnetons). These spins, located at $z=0$ and with lattice spacing $a$, represent the magnetic moments of single atoms. They can change their orientation but not their absolute value, so that there are two degrees of freedom per spin. We use open boundary conditions. A constant point dipole $\mathbf{S}_{\text {tip }}$ pointing in the $z$-direction and located at $z=2 a$ represents the magnetic tip. It is moved parallel to the surface with constant velocity $\mathbf{v}$.

This model has only magnetic degrees of freedom and thus focusses on their contributions to friction. For a real tip one could expect that magnetic, just like 
nonmagnetic $[11,12]$ interactions might also lead to atomic stick-slip behaviour, and hence to phononic dissipation with a velocity-independent friction contribution as described by the Prandtl-Tomlinson model $[13,14]$. However, this requires a periodic potential between tip and substrate, that is strong enough compared to the elastic deformation energy to allow for multiple local potential energy minima. The magnetic tip-substrate interactions are unlikely to be strong enough.

The Hamiltionan consists of two parts:

$$
\mathcal{H}=\mathcal{H}_{\text {sub }}+\mathcal{H}_{\text {sub-tip }}
$$

The first one represents the internal ferromagnetic shortrange interaction within the substrate. The second one describes the long-range coupling between the substrate and the tip.

The interaction between the substrate moments is modeled by the anisotropic classical Heisenberg model,

$$
\mathcal{H}_{\mathrm{sub}}=-J \sum_{\langle i, j\rangle} \mathbf{S}_{i} \cdot \mathbf{S}_{j}-d_{z} \sum_{\mathrm{i}=1}^{N} S_{i, z}^{2} .
$$

$J>0$ describes the ferromagnetic exchange interaction between two nearest neighbours, expressed by the angular brackets $\langle i, j\rangle . d_{z}<0$ quantifies the anisotropy, which prefers in-plane orientations of the spins. The dipoledipole interaction between the substrate spins is neglected, because it is much weaker than the exchange interaction. A quantitative comparison of our simulation results with the ones obtained in [8], where the dipole-dipole interaction inside the substrate was taken into account, justifies this approximation, which reduces simulation time enormously.

The long-range interaction between substrate and tip is described by a dipole-dipole interaction term

$$
\mathcal{H}_{\text {sub-tip }}=-w \sum_{i=1}^{N} \frac{3\left(\mathbf{S}_{i} \cdot \mathbf{e}_{i}\right)\left(\mathbf{S}_{\text {tip }} \cdot \mathbf{e}_{i}\right)-\mathbf{S}_{i} \cdot \mathbf{S}_{\text {tip }}}{R_{i}^{3}},
$$

where $R_{i}=\left|\mathbf{R}_{i}\right|$ denotes the norm of the distance vector $\mathbf{R}_{i}=\mathbf{r}_{i}-\mathbf{r}_{\text {tip }}$, and $\mathbf{e}_{i}$ its unit vector $\mathbf{e}_{i}=\mathbf{R}_{i} / R_{i} \cdot \mathbf{r}_{i}$ and $\mathbf{r}_{\text {tip }}$ denote the position vectors of the substrate spins and the tip, respectively. $w$ quantifies the dipole-dipole coupling of the substrate and the tip. Note, however, that the results of the present study only depend on the combination $w\left|\mathbf{S}_{\text {tip }}\right|$, which is the true control parameter for the tipsubstrate coupling.

The proper equation of motion of the magnetic moments is the Landau-Lifshitz-Gilbert (LLG, [15]) equation,

$$
\frac{\partial}{\partial t} \mathbf{S}_{i}=-\frac{\gamma}{\left(1+\alpha^{2}\right) \mu_{s}}\left[\mathbf{S}_{i} \times \mathbf{h}_{i}+\alpha \mathbf{S}_{i} \times\left(\mathbf{S}_{i} \times \mathbf{h}_{i}\right)\right],
$$

which is equivalent to the Gilbert equation of motion [16]:

$$
\frac{\partial}{\partial t} \mathbf{S}_{i}=-\frac{\gamma}{\mu_{s}} \mathbf{S}_{i} \times\left[\mathbf{h}_{i}-\frac{\alpha \mu_{s}}{\gamma} \frac{\partial \mathbf{S}_{i}}{\partial t}\right]
$$

The first term on the right-hand side of eq. (4) describes the dissipationless precession of each spin in the effective field $\mathbf{h}_{i}$ (to be specified below). The second term describes the relaxation of the spin towards the direction of $\mathbf{h}_{i}, \gamma$ denotes the gyromagnetic ratio (for free electrons $\gamma=1.76086 \times 10^{11} \mathrm{~s}^{-1} \mathrm{~T}^{-1}$ ), and $\alpha$ is a phenomenological, dimensionless damping parameter.

The effective field contains contributions from the tip and from the exchange interaction, as well as a thermally fluctuating term $\zeta_{i}[17,18]$,

$$
\mathbf{h}_{i}=-\frac{\partial \mathcal{H}}{\partial \mathbf{S}_{i}}+\boldsymbol{\zeta}_{i}(t)
$$

The stochastic, local and time-dependent vector $\zeta_{i}(t)$ expresses a "Brownian rotation", which is caused by the heat-bath connected to each magnetic moment. In our simulations this vector is realised by uncorrelated random numbers with a Gaussian distribution, which satisfy the relations

$$
\left\langle\zeta_{i}(t)\right\rangle=0
$$

and

$$
\left\langle\zeta_{i}^{\kappa}(t) \zeta_{j}^{\lambda}\left(t^{\prime}\right)\right\rangle=2 \frac{\alpha \mu_{s}}{\gamma} k_{B} T \delta_{i, j} \delta_{\kappa, \lambda} \delta\left(t-t^{\prime}\right),
$$

where $T$ is the temperature, $\delta_{i, j}$ expresses that the noise at different lattice sites is uncorrelated, and $\delta_{\kappa, \lambda}$ refers to the absence of correlations among different coordinates.

To find a quantity which expresses the friction occurring in the system, it is helpful to discuss energy transfers between tip, substrate and heat-bath first. It is straightforward to separate the time derivative of the system energy, eq. (1), into an explicit and an implicit one,

$$
\frac{\mathrm{d} \mathcal{H}}{\mathrm{d} t}=\frac{\partial \mathcal{H}}{\partial t}+\sum_{i=1}^{N} \frac{\partial \mathcal{H}}{\partial \mathbf{S}_{i}} \cdot \frac{\partial \mathbf{S}_{i}}{\partial t} .
$$

The explicit time dependence is exclusively due to the tip motion. The energy transfer between the tip and the substrate is expressed by the first term of eq. (9), which justifies to call it the "pumping power" $\mathcal{P}_{\text {pump }}$

$$
\begin{aligned}
& \mathcal{P}_{\text {pump }}=\frac{\partial \mathcal{H}}{\partial t}=\frac{\partial \mathcal{H}_{\text {sub-tip }}}{\partial \mathbf{r}_{\mathrm{tip}}} \cdot \mathbf{v}= \\
& w \sum_{\alpha} v_{\alpha} \sum_{i=1}^{N} \frac{3}{R_{i}^{4}}\left\{\left(\mathbf{S}_{i} \cdot \mathbf{e}_{i} e_{i, \alpha}-S_{i, \alpha}\right)\left(\mathbf{S}_{\mathrm{tip}} \cdot \mathbf{e}_{i}\right)\right. \\
& +\left(\mathbf{S}_{\mathrm{tip}} \cdot \mathbf{e}_{i} e_{i, \alpha}-S_{\mathrm{tip}, \alpha}\right)\left(\mathbf{S}_{i} \cdot \mathbf{e}_{i}\right) \\
& \left.-\mathbf{S}_{i} \cdot \mathbf{S}_{\mathrm{tip}} e_{i, \alpha}+3 e_{i, \alpha}\left(\mathbf{S}_{i} \cdot \mathbf{e}_{i}\right)\left(\mathbf{S}_{\mathrm{tip}} \cdot \mathbf{e}_{i}\right)\right\}
\end{aligned}
$$

At any instance, the substrate exerts a force $-\frac{\partial \mathcal{H}_{\text {sub-tip }}}{\partial r_{\text {tip }}}$ on the tip. Due to Newton's third law, $\mathcal{P}_{\text {pump }}$ is the work per unit time done by the tip on the substrate. Its time and thermal average $\left\langle\mathcal{P}_{\text {pump }}\right\rangle$ is the average rate at which energy is pumped into the spin system. In a steady state 
it must be equal to the average dissipation rate, i.e. to the net energy transferred to the heat bath per unit time due to spin relaxation. The magnetic friction force can therefore be calculated by

$$
F=\frac{\left\langle\mathcal{P}_{\text {pump }}\right\rangle}{v} \text {. }
$$

The second term of eq. (9) describes the energy transfer between the spin system and the heat bath. Inserting eq. (5) into $\mathcal{P}_{\text {diss }}=-\sum_{i=1}^{N} \frac{\partial \mathcal{H}}{\partial \mathbf{S}_{i}} \cdot \frac{\partial \mathbf{S}_{i}}{\partial t}$ leads to

$$
\begin{aligned}
\mathcal{P}_{\text {diss }} & =\sum_{i=1}^{N} \frac{\partial \mathcal{H}}{\partial \mathbf{S}_{i}} \cdot\left[\frac{\gamma}{\mu_{s}} \mathbf{S}_{i} \times \zeta_{i}-\alpha \mathbf{S}_{i} \times \frac{\partial \mathbf{S}_{i}}{\partial t}\right] \\
& =-\mathcal{P}_{\text {therm }}+\mathcal{P}_{\text {relax }} .
\end{aligned}
$$

The first term, $\mathcal{P}_{\text {therm }}$ containing $\zeta_{i}$, describes, how much energy is transferred into the spin system due to the thermal perturbation by the heat bath. The second term, $\mathcal{P}_{\text {relax }}$ proportional to the damping constant $\alpha$, describes the rate of energy transfer into the heat bath due to the relaxation of the spins.

At $T=0, \mathcal{P}_{\text {therm }}$ is zero. The spins are only perturbed by the external pumping at $v \neq 0$. Then

$$
\mathcal{P}_{\text {relax }}=\mathcal{P}_{\text {diss }}=\frac{\gamma \alpha}{\mu_{s}\left(1+\alpha^{2}\right)} \sum_{i=1}^{N}\left(\mathbf{S}_{i} \times \mathbf{h}_{i}\right)^{2}, \quad(T=0),
$$

where for the last transformation we used eq. (4) in order to show explicitly the relationship between dissipation rate and misalignment between spins and local fields.

It will be instructive to compare the magnetic substrate scanned by a dipolar tip with a much simpler system, in which the substrate is replaced by a single spin $\mathbf{S}$ subjected to an external field $\mathbf{h}(t)$ that rotates in the plane perpendicular to a constant angular velocity $\omega$ (replacing the tip velocity). It is straightforward to obtain the steady state solution for $T=0$, where in the co-rotating frame $\mathbf{S}$ is at rest. $\mathbf{S}$ lags behind $\mathbf{h} / h$ by an angle $\delta \varphi$ and gets a component $\delta \theta$ in $\boldsymbol{\omega}$-direction (cf. fig. 1), which are in first order given by

$$
\frac{\delta \varphi}{\alpha}=\delta \theta=\frac{\omega \mu_{s}}{h \gamma}+O\left(\left(\frac{\omega \mu_{s}}{h \gamma}\right)^{3}\right)
$$

Inserting this into the $(N=1)$-case of eq. (13) yields a dissipation rate of $\mathcal{P}_{\text {diss }}=\alpha \omega^{2} \mu_{s} / \gamma$, which corresponds to a "viscous" friction $F=\mathcal{P}_{\text {diss }} / \omega \propto \alpha \omega$.

It is instructive to give a simple physical explanation for eq. (14), instead of presenting the general solution, which can be found in [19]. Two timescales exist in the system, which can be readily obtained from eq. (4); first the inverse Lamor frequency $\tau_{\text {Lamor }}=\left(1+\alpha^{2}\right) \mu_{s} / \gamma h$, and second, the relaxation time $\tau_{\text {relax }}=\tau_{\text {Lamor }} / \alpha$. They govern the time evolution of $\delta \varphi$ and $\delta \theta$. In leading order,

$$
\delta \dot{\theta}=\frac{\delta \varphi}{\tau_{\text {Lamor }}}-\frac{\delta \theta}{\tau_{\text {relax }}}
$$

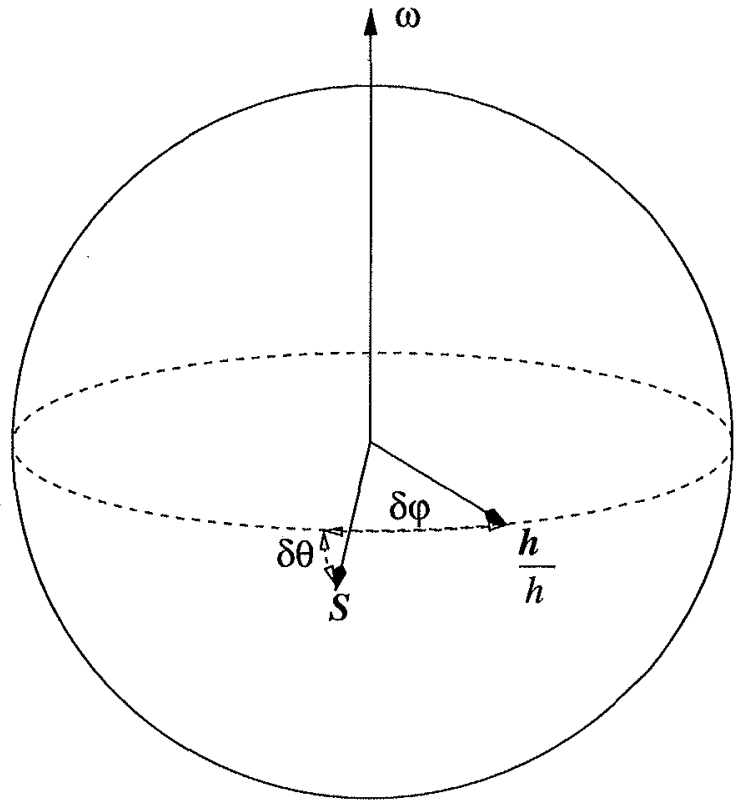

Fig. 1: A single spin in a magnetic field rotating with angular velocity $\omega$, is dragged along with a phase shift $\delta \varphi$ and aquires an out-of-plane component $\delta \theta$.

$$
\delta \dot{\varphi}=\omega-\frac{\delta \theta}{\tau_{\text {Lamor }}}-\frac{\delta \varphi}{\tau_{\text {relax }}} .
$$

The first equation describes, how $\delta \theta$ would increase by precession of the spin around the direction of the field, which is counteracted by relaxation back towards the equator. The second equation describes that without relaxation into the field direction, $\delta \varphi$ would increase with velocity $\omega$ minus the azimuthal component of the precession velocity, which is in leading order proportional to $\delta \theta$. Setting the left hand sides to zero in the steady state, immediately gives the solution (14).

In the $(T=0)$ study [8], the time average $\left\langle\mathcal{P}_{\text {relax }}\right\rangle / v$ was used to calculate the friction force. As pointed out above, this quantity agrees with (11) in the steady state. For finite temperatures, however, (11) is numerically better behaved than $\left\langle\mathcal{P}_{\text {relax }}\right\rangle / v$. The reason is the following:

For $T \neq 0$, the spins are also thermally agitated, even without external pumping, when the dissipation rate $\mathcal{P}_{\text {diss }}$ vanishes. This shows that the two terms $\mathcal{P}_{\text {therm }}$ and $\mathcal{P}_{\text {relax }}$ largely compensate each other, and only their difference is the dissipation rate we are interested in. This fact makes it difficult to evaluate (12) and is the reason why we prefer to work with (11) as the definition of the friction force.

We have also analyzed the fluctuations of the friction force (11). The power spectrum has a distinct peak at frequency $v / a$, which means that the dominant temporal fluctuations are due to the lattice periodicity with lattice constant $a$. A more complete investigation of the fluctuations, which should also take into account, how thermal 
positional fluctuations influence the friction force, remains to be done.

Technical remarks. - Because of the vector product in eq. (4), the noise $\zeta_{i}$ enters in a multiplicative way, calling for special attention to the interpretation of this stochastic differential equation (Stratonovich vs. Itô sense). The physical origin of the noise renders it generically coloured and thus selects the Stratonovich interpretation as the appropriate one (Wong-Zakai theorem [20]), in which its appearance as white noise is an idealisation. Accordingly we employ the Heun integration scheme [21]. After each time step the spins are rescaled so that their length remains unchanged.

To get meaningful results, it is of prime importance to reach a steady state. The initial configuration turned out to be a crucial factor for achieving this within acceptable computing time. Therefore a long initialisation run is performed, before the tip motion starts.

Moreover, the system size is another limiting factor. In order to avoid that the tip reaches the system boundary before the steady state is reached, we use a "conveyor belt method" allowing to do the simulation in the comoving frame of the tip. The tip is placed in a central point above the substrate plane, e.g. at $\left(\left(L_{x}+1\right) / 2,\left(L_{y}+1\right) / 2\right)$. After an equilibration time, the tip starts to move with fixed velocity in the $x$-direction. When it passed exactly one lattice constant $a$, the front line (at $x=L_{x}$ ) is duplicated and added to the lattice at $x=L_{x}+1$. Simultaneously, the line at the opposite boundary of the system (at $x=1$ ) is deleted, so that the simulation cell is of fixed size and consists of the $L_{x} \times L_{y}$ spins centered around the tip position, with open boundaries. Note that this is different from periodic boundary conditions, because the spin configuration deleted at one side is different from the one added at the opposite side. We compared the results obtained for a small system in the co-moving frame of the tip with some runs for a system that was long enough in the $x$-direction that the steady state could be reached in the rest frame of the sample, and confirmed that the same friction results and steady state properties could be obtained with drastically reduced computation time.

It is convenient to rewrite the equations of motion in natural units. An energy unit is prescribed by the exchange energy $J$ of two magnetic moments. It rescales the energy related parameters $d_{z}$ and $w$ as well as the simulated temperature,

$$
k_{B} T^{\prime}=\frac{k_{B} T}{J}
$$

The rescaled time further depends on the material constants $\mu_{s}$ and $\gamma$,

$$
t^{\prime}=\frac{J \gamma}{\mu_{s}} t
$$

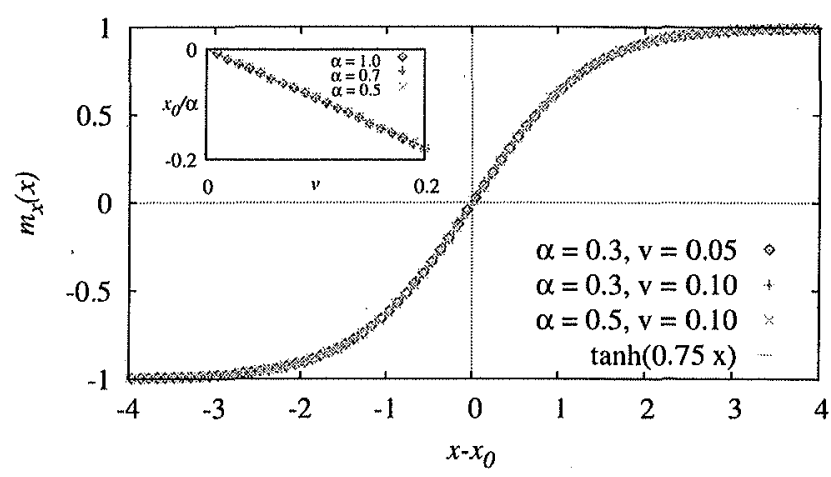

Fig. 2: Local magnetisation ( $x$-component) at $T=0$ along the lattice axes in the $x$-direction which are closest to the tip (i.e. at $y= \pm 0.5$ ). Analogous to a domain wall one finds a $\tanh x$ profile. Depending on the damping constant $\alpha$ and the velocity $v$, its zero is shifted backwards from the tip position by a value $x_{0} \approx-0.88 \alpha v$, as shown in the inset.

A length scale is given by the lattice constant $a$, so a natural velocity for the system can be defined,

$$
v^{\prime}=\frac{\mu_{s}}{\gamma J a} v
$$

From the natural length and energy, the natural force results to:

$$
F^{\prime}=\frac{a}{J} F
$$

From now on, all variables are given in natural units, and we will drop the primes for simplicity. The typical extension of the simulated lattices is $50 \times 30$, which was checked to be big enough to exclude finite-size effects. For the tip coupling, we chose large values $\left(e . g . w \mathbf{S}_{\text {tip }}=(0,0,-10)\right)$, to get a large effective field on the substrate. Usually it is assumed that the dipole-dipole coupling constant has a value of about $w=0.01$, which means that the magnetic moment of the tip is chosen a factor of 1000 times larger than the individual substrate moments. The anisotropy constant is set to $d_{z}=-0.1$ in all simulations. The damping constant $\alpha$ is varied from 0.1 to the quite large value 1. At finite temperatures typically 50 simulation runs with different random number seeds are performed to get reliable ensemble averages.

Simulation results. - In [8] it was found that the magnetic friction force depends linearly on the scanning velocity $v$ and the damping constant $\alpha$ for small velocities $(v \leq 0.3)$. For higher velocities the friction force reaches a maximum and then decreases. In this work we focus on the low-velocity regime with the intention to shed more light on the friction mechanism and its temperature dependence ${ }^{1}$.

\footnotetext{
${ }^{1}$ It should be noted that the smallest tip velocities we simulated, are of the order of $10^{-2}\left(a J \gamma / \mu_{s}\right)$, which is still fast compared to typical velocities in friction force microscopy experiments.
} 

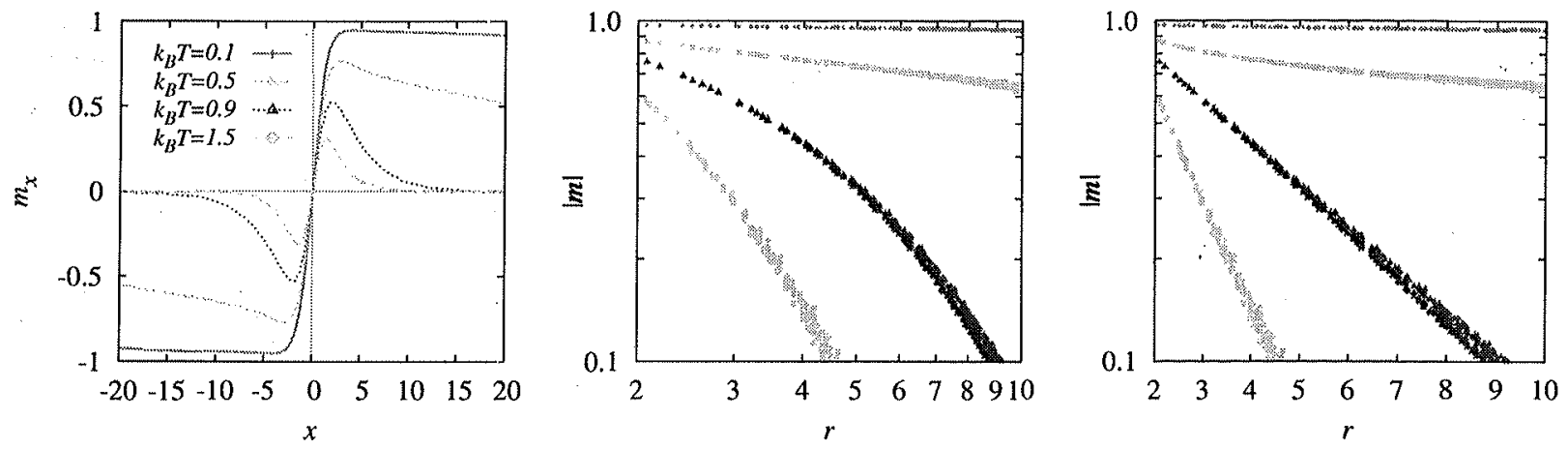

Fig. 3: Left: magnetisation profiles as in fig. 2 for several temperatures with $w S_{\text {tip }}=10, \alpha=0.5$ and $v=0.01$. Middle and right: absolute value of the average magnetisation as a function of the distance $r$ from $(x, y)=(0,0)$, directly underneath the tip. For small temperatures (upper two curves) it decreases with a power law (cf. double-logarithmic plot, middle), for high temperatures (lower two curves) it decreases exponentially (cf. semi-logarithmic plot, right).

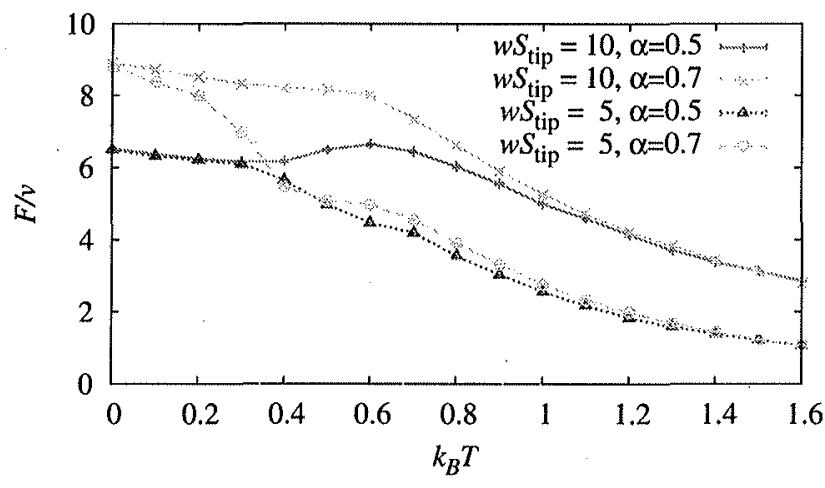

Fig. 4: Friction coefficients for different $\alpha, w S_{\text {tip }}$ and $k_{B} T$. One can distinguish between a low-temperature regime, where the friction coefficient depends on $\alpha$ but not on $w S_{\text {tip }}$, and a hightemperature regime, where it depends on $w S_{\text {tip }}$ but not on $\alpha$.

Adiabatic approximation at $T=0$. If we assume the field $\mathbf{h}$ for each spin to vary slowly enough to allow the solution (14) to be attained as adiabatic approximation, the linear dependence $F \propto \alpha v$ from [8] follows immediately: At every point, the temporal change of the direction of $\mathbf{h}$, defining a local $\omega$ for (14), is proportional to $v$. We confirmed the validity of the adiabatic approximation numerically by decomposing $\mathbf{S}-\mathbf{h} / h$ with respect to the local basis vectors $\partial_{t}(\mathbf{h} / h), \mathbf{h}$, and their crossproduct, all of them appropriately normalised. In other words, we extracted $\delta \varphi$ and $\delta \theta$ directly and found them in excellent agreement with (14).

The lag of $\mathbf{S}$ with respect to $\mathbf{h}$ manifests itself also macroscopically in the magnetisation field as we will show now. The tip-dipole is strong enough to align the substrate spins to nearly cylindrical symmetry. Since the anisotropy is chosen to generate an easy plane $\left(d_{z}<0\right)$, spins far away from the tip try to lie in the $x y$-plane, while close to the tip they tilt into the $z$-direction. This is displayed in fig. 2 where the $x$-component of the local magnetisation is shown along a line in the $x$-direction for a fixed

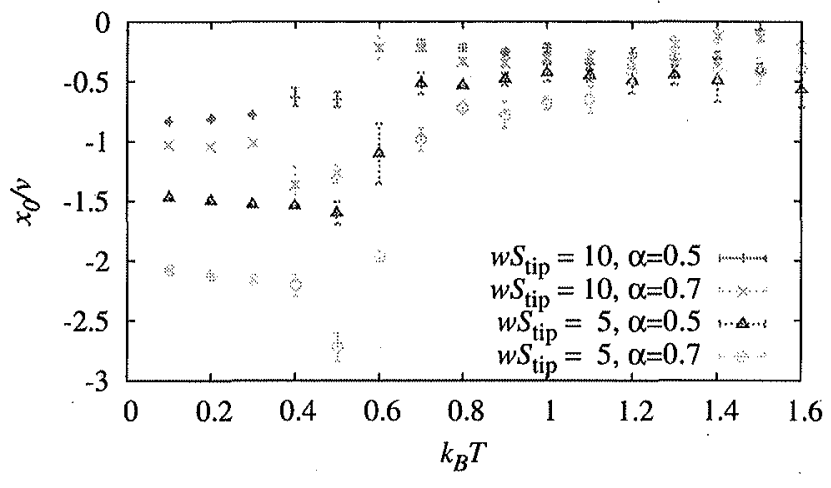

Fig. 5: Distance $x_{0}$ by which the magnetisation pattern lags behind the tip is proportional to $v$ for all temperatures. The proportionality constant depends on $\alpha$ only in the lowtemperature regime.

$y$-coordinate. Remarkably, these magnetisation profiles for different values of $v$ and $\alpha$ collapse onto a unique curve, if they are shifted by corresponding offsets $x_{0}$ with respect to the tip position. As expected from (14), the magnetisation profile stays behind the tip by a ( $y$-dependent) shift $x_{0} \propto$ $\alpha v$ (cf. inset of fig. 2).

Friction at $T>0$. With increasing temperature the magnetisation induced by the tip becomes smaller, as shown in fig. 3. One can distinguish a low-temperature regime, where the local magnetisation decreases algebraically with the distance from the tip, and a hightemperature regime, where it decreases exponentially. The transition between these regimes happens around $T \approx 0.7$.

For all temperatures the friction force $F$ turns out to be proportional to the velocity (up to $v \approx 0.3$ ), as for $T=0$, with a temperature-dependent friction coefficient $F / v$.

The two temperature regimes manifest themselves also here, as shown in fig. 4: For low temperatures the friction coefficients depend nearly linearly on $\alpha$, reflecting the $T=0$ behaviour. Towards the high-temperature regime, however, the $\alpha$-dependence vanishes, and all friction 
coefficients merge into a single exponentially decreasing function.

The low-temperature behaviour can be understood essentially along the lines worked out for $T=0$, as result of a delayed, deterministic response (precession and relaxation) to the time-dependent tip field. At high temperatures, however, friction results from the ability of the tip to propagate partial order through the thermally disordered medium. The magnetisation pattern in the wake of the tip no longer adapts adiabatically to the dwindling influence of the tip, but decays due to thermal disorder. Then, a rising temperature lets the ordered area around the tip shrink which leads to the exponential decrease of the friction coefficient. However, it increases with the tip strength, $w S_{\text {tip }}$, as stronger order can be temporarily forced upon the region around the tip. By contrast, the tip strength looses its influence on friction in the limit $T \rightarrow 0$, because the substrate spins are maximally polarised in the tip field.

This picture of the two temperature regimes is supported by the distance $x_{0}$, by which the magnetisation pattern lags behind the tip. It is proportional to $v$ for all temperatures, but $\alpha$-dependent only in the low-temperature regime, cf. fig. 5 .

Conclusion and outlook. - In this work, we could explain the low-velocity, zero-temperature findings from [8], namely that the magnetic friction force in the Heisenberg model has a linear velocity dependence with a coefficient proportional to the damping constant $\alpha$. In the spin polarisation cloud dragged along with the tip, each substrate spin follows the local field with a lag proportional to the frequency of the field change and to $\alpha$. Moreover, the magnetisation pattern around the tip gets distorted due to precession. These effects directly give rise to the observed magnetic friction and could be evaluated quantitatively by mears of a single spin model.

Second, for the first time the temperature dependence of magnetic friction in the Heisenberg model was investigated in the framework of Landau-Lifshitz-Gilbert (LLG) dynamics with a stochastic contribution to the magnetic field. Two regimes were found, which can be characterised by their different relaxation behaviour. While in the low-temperature regime the response of the system on the perturbation due to the moving tip is dominated by the deterministic precession and relaxation terms in the LLG equation, thermal perturbations competing with the one caused by the moving tip are essential in the hightemperature regime. This explains, why magnetic friction depends on $\alpha$ but not on $w S_{\text {tip }}$ for low temperatures, while it depends on $w S_{\text {tip }}$ but not on $\alpha$ for high temperatures where it decreases exponentially with $T$.

Important extensions of the present investigation include the effects of a tip magnetisation pointing in a different than the $z$-direction, of the strength and sign of spin anisotropy, $d_{z}$, or of the thickness of the magnetic layer. Both, spin anisotropy and lattice dimension will be crucial for the critical behaviour, as well as for the critical temperature itself. Studies dealing with these quantities are already in progress and will be reported in a future work.

This work was supported by the German Research Foundation (DFG) via SFB 616 "Energy dissipation at surfaces". Computation time was granted in Jülich by the John-von-Neumann Institute of Computing (NIC).

\section{REFERENCES}

[1] PERsson B., Sliding Friction (Springer, Berlin, Heidelberg, New York) 1998.

[2] URbakH M., Klafter J., Gourdon D. and ISRAELACHVILI J., Nature, 430 (2004) 525.

[3] Gnecco E., Bennewitz R., Gyalog T. and Meyer E., J. Phys.: Condens. Matter, 13 (2001) R619.

[4] Acharyya M. and Chakrabarti B. K., Phys. Rev. B, 52 (1995) 6550

[5] Ortín J. and Gorcoechea J., Phys. Rev. B, 58 (1998) 5628.

[6] Corberi F., Gonnella G. and lamura A., Phys. Rev. Lett., 81 (1998) 3852.

[7] Kadau D., Hucht A. and Wolf D. E., Phys. Rev. Lett, 101 (2008) 137205.

[8] Fusco C., Wolf D. E. and Nowak U., Phys. Rev. B, 77 (2008) 174426.

[9] Grutter P., Liu Y., LeBlanc P. and Durig U., Appl. Phys. Lett., 71 (1997) 279.

[10] Schmidt R., Lazo C., Holscher H., Pi U. H., Caciuc V., Schwarz A., Wiesendanger R. and Heinze S., Nano Lett., 9 (2009) 200.

[11] ZwÖrner O., Hölscher H., Schwarz U. D. and Wiesendanger R., Appl. Phys. A, 66 (1998) S263.

[12] Gnecco E., Bennewitz R., Gyalog T., Loppacher C., Bammerlin M., Meyer E. and Güntherodt H.-J., Phys. Rev. Lett., 84 (2000) 1172.

[13] Prandtl L., Z. Angew. Math. Mech., 8 (1928) 85.

14] Tomlinson G. A., Philos. Mag., 7 (1929) 905.

[15]. Landau L. D. and Lifshitz E. M., Phys. Z. Sowjetunion, 8 (1935) 153.

[16] GilberT T. L., IEEE Trans. Magn., 40 (2004) 3443.

[17] Néel L., C. R. Acad. Sci. Paris, 228 (1949) 664.

[18] Brown W. F., Phys. Rev., 130 (1963) 1677.

[19] MagierA M. P., Computer Simulation of Magnetic Friction, Diploma Thesis, University of Duisburg-Essen (2008).

[20] Horsthemke W. and Lefever R., Noise-Induced Transitions (Springer) 1983.

[21] García-Palacios J. L. and Lázaro F. L., Phys. Rev. $B, 58(1998) 14937$. 